\title{
Pour une interdisciplinarité «focalisée » dans les sciences humaines et sociales
}

\section{Patrick Charaudeau}

\section{(2) OpenEdition \\ 1 Journals}

Édition électronique

URL : http://journals.openedition.org/questionsdecommunication/385

DOI : 10.4000/questionsdecommunication.385

ISSN : 2259-8901

\section{Éditeur}

Presses universitaires de Lorraine

\section{Édition imprimée}

Date de publication : 30 juin 2010

Pagination : 195-222

ISBN : 978-2-8143-0024-8

ISSN : 1633-5961

\section{Référence électronique}

Patrick Charaudeau, «Pour une interdisciplinarité «focalisée » dans les sciences humaines et sociales ", Questions de communication [En ligne], 17 | 2010, mis en ligne le 24 août 2015, consulté le 10 décembre 2020. URL : http://journals.openedition.org/questionsdecommunication/385; DOI : https://doi.org/10.4000/questionsdecommunication.385

Ce document a été généré automatiquement le 10 décembre 2020.

Tous droits réservés 


\title{
Pour une interdisciplinarité «focalisée » dans les sciences humaines et sociales
}

\author{
Patrick Charaudeau
}

\section{NOTE DE L'ÉDITEUR}

Dans la prochaine livraison de Questions de communication, la contribution de Patrick Charaudeau sera discutée par plusieurs chercheurs.

1 Comment prétendre traiter une question aussi vaste et complexe que l'interdisciplinarité quand, d'un côté, Claude Lévi-Strauss $(1958$; 1962) invitait, au nom de la méthode du bricolage, à établir des connexions entre l'anthropologie, la linguistique, la littérature, l'art, la psychologie, le droit, la religion, etc., de l'autre, Edgar Morin incite, au-delà même de la transdisciplinarité, à « écologiser les disciplines » en tenant compte de « tout ce qui est contextuel y compris des conditions culturelles et sociales » et en adoptant parfois un point de vue «métadisciplinaire »; et de citer Pascal (in : Kourilsky, 1990) qui, selon lui, en aurait été le précurseur: "Toutes choses étant causées et causantes, aidées et aidantes, médiates et immédiates, et toutes s'entretenant par un lien naturel et insensible qui lie les plus éloignées et les plus différentes, je tiens impossible de connaitre les parties sans connaître le tout, non plus que de connaître le tout sans connaître particulièrement les parties »? Il semble cependant qu'il est une attitude, à la fois plus modeste et non dépourvue d'intérêt, qui consiste à interroger la notion même d'interdisciplinarité à la lumière de certains travaux qui s'en réclament, et - si l'on peut leur accorder quelque crédit - de mes propres travaux qui ont toujours défendu et pratiqué une telle perspective d'analyse. Il ne s'agit donc pas d'embrasser la totalité des disciplines des sciences humaines et sociales. Je n'aborderai pas cette question du point de vue des champs disciplinaires institutionnellement définis dont on sait, depuis les travaux de Pierre Bourdieu qu'ils sont aussi des « champs de pouvoir ». Ce ne sera pas davantage en 
examinant les théories et leurs méthodologies les unes après les autres pour dresser un constat d'interconnexions entre certaines disciplines, comme cela est l'objet de certains colloques ou ouvrages collectifs (voir par exemple Bonnafous, Temmar, 2007). On n'entrera pas non plus dans la polémique qui a opposé, dans les années 70-80, les tenants d'une monodisciplinarité à ceux d'une pluridisciplinarité. Il était alors délicat de parler d'interdisciplinarité, de pluridisciplinarité ou de transdisciplinarité, car l'on courrait le risque de se voir dénier le label de scientificité par ceux qui estimaient que seule la monodisciplinarité pouvait prétendre à cette qualification. La rigueur de la démarche scientifique exigerait une grande spécialisation, elle ne pourrait s'exercer que dans un territoire conceptuel bien circonscrit autour de postulats et/ou hypothèses bien déterminés, avec des outils d'analyse propres à chaque discipline. Pourtant, parallèlement, les penseurs et chercheurs de l'époque, tels Claude Lévi-Strauss, Roland Barthes, Michel Foucault, Edgar Morin, pour ne citer qu'eux ${ }^{1}$, n'ont eu de cesse de naviguer entre différentes disciplines, sans cependant théoriser une quelconque interdisciplinarité. Était revendiquée une inter-, pluri- ou trans-disciplinarité au nom de la complexité croissante du monde, de l'éclatement de la connaissance, de la pluralité des savoirs sur des mêmes faits sociaux et de leur nécessaire articulation. Cette opposition renforcée par le jeu institutionnel de défense d'un territoire disciplinaire à des fins de visibilité sociale et de constitution d'un savoir académique qui doit faire l'objet d'un enseignement dans le cadre d'un système scolaire et universitaire - a instauré une rivalité de fait entre chercheurs des sciences humaines et sociales, les uns reprochant à d'autres de ne pas se situer dans le noyau dur de la discipline, seul garant, d'après eux, de la rigueur scientifique, et de préférer la périphérie molle $^{2}$, les autres critiquant l'enfermement des gardiens du monodisciplinaire, car, d'après eux, ceux-ci ne peuvent voir qu'un aspect très partiel des phénomènes humains à travers des micro-analyses, lesquelles, sans que l'on en nie l'intérêt, semblent ne servir qu'elles-mêmes et font obstacles à la compréhension globale des phénomènes étudiés. À ce type d'antagonisme, il faut ajouter le phénomène d'«ostracisation" de certains courants théoriques au détriment d'autres. Au nom de l'adhésion à de nouveaux paradigmes qui se voulaient plus explicatifs que les précédents, s'imposaient des modes de pensée et d'analyse tendant à prendre toute la place du champ scientifique (le fonctionnalisme, le structuralisme, le pragmatisme) au point parfois de prononcer des excommunications, comme ce fut le cas avec le marxisme ou le lacanisme, pour ne citer que ceux-là, et ce pour des raisons plus idéologiques que scientifiques. Ces oppositions n'ont pas tout à fait disparu. Certes, il n'est plus de discipline pouvant se prévaloir d'une omnipotence qui lui ferait croire qu'elle domine de façon absolue le champ des sciences humaines et sociales. Mais des oppositions demeurent avec des atours différents. D'un côté, des chercheurs ayant recours à de nouveaux modes d'analyse, à l'aide d'outils technologiques qu'ils donnent comme principe d'explication (par exemple, le traitement informatique de corpus permettant d'établir de savants résultats statistiques), alors que, d'après leurs opposants, ce ne sont que des nouveaux modes de recueil, d'accumulation et d'ordonnancement des données empiriques; ou bien les tenants d'analyses cognitives, voire neuronales, donnant comme horizon explicatif ce qui, in fine, fonctionnerait dans le cerveau humain. D'un autre côté, les partisans d'analyses qualitatives détaillées et de démarches interprétatives se situant au carrefour de différentes disciplines, comme en témoignent, au-delà même des sciences humaines et sociales, certains projets de recherche entre sciences biologiques et sciences sociologiques ${ }^{3}$. En effet, on voit fleurir des colloques et des ouvrages consacrés à la question de la pluridisciplinarité, manifestations qui, au nom de 
la complexité croissante du savoir, témoignent du souci de faire se rencontrer différentes disciplines. Ce n'est pas de cette façon polémique que j'aborderai ici la question de l'interdisciplinarité. Je prendrai plutôt le parti d'une interrogation sur la possible rencontre de disciplines. Mais si une telle rencontre s'impose, comment celle-ci doit-elle s'opérer ? J'ai écarté l'option qui consiste à comparer des disciplines institutionnellement définies, car on risque de tomber dans une discussion stérile sur l'importance de chacune d'elles. J'ai également écarté la démarche qui consiste à examiner les théories en ellesmêmes, car on risque de se voir reprocher l'incompréhension de telle théorie particulière, si «l'on n'est pas dedans $»^{4}$. Doit-elle alors se faire en confrontant des concepts fondateurs, en examinant les outils d'analyse, ou doit-on observer comment différentes disciplines analysent un même objet d'étude ? Cette dernière option semble se prêter davantage à une rencontre des disciplines, mais il n'est guère envisageable de les passer toutes en revue, les unes après les autres, travail considérable auquel s'est attelé Edgar Morin. Ce que je voudrais faire ici, plus modestement, c'est aborder cette question par le biais de quelques problématisations qui sont susceptibles de rendre compte de la façon dont peuvent être décrits les phénomènes sociaux à travers des pratiques analysantes. M'interrogeant d'abord sur ce qui constitue une discipline, je voudrais tenter de pointer ce que devraient être les conditions d'une interdisciplinarité, et montrer comment celle-ci peut être mise en œuvre dans le champ de l'analyse des phénomènes de communication. À ce projet, un dernier élément : cette exploration sera faite du point de vue d'un sémiologue, analyste du discours, qui, ayant traversé les sciences du langage de l'époque structuraliste à nos jours, a eu à se confronter aux réflexions et travaux de sémanticiens autour de Bernard Pottier, de sémioticiens autour d'Algirdas Greimas, de narratologues autour de Gérard Genette, d'une certaine philosophie autour de Michel Foucault, d'une sémiologie polyvalente autour de Roland Barthes ${ }^{5}$, et a été conduit à collaborer avec des sociologues et des psychosociologues lors de travaux portant sur les médias et la communication, sans parler des philosophes du politique lors des analyses sur le discours politique. Il est donc possible que des chercheurs ayant eu d'autres itinéraires ne partagent pas certains de mes points de vue. De plus, ils pourront juger que, dans l'effort qui est le mien de trouver des notions communes à plusieurs disciplines, je fais cavalièrement l'impasse sur des distinctions plus fines qui montreraient au bout du compte qu'il y a plus de différences que de similitudes, mais c'est une condition de l'esprit d'interdisciplinarité que d'accepter certaines simplifications (et non déformations) pour établir des ponts entre les disciplines. Pour ceux qui seraient heurtés par une telle démarche, c'est précisément à un échange de points de vue que je les invite.

\section{Une première difficulté : l'usage des termes}

2 Comme bien souvent dans l'histoire des sciences humaines et sociales, il y a des moments de modes. Mais une mode, pour qu'elle s'impose, a besoin d'être déclarée comme telle, même si on ne l'applique pas de façon systématique. Elle est déclarée de diverses façons : ici, de manière institutionnelle, lorsque, par exemple, dans le projet de constitution d'un "pôle de recherche » on décrète dans la charte intellectuelle de création d'un campus universitaire que celui-ci instaurera une "synergie» entre différentes disciplines permettant une "interdisciplinarité effective " ${ }^{6}$; là, dans l'appel d'offre d'un colloque dont le titre est «Discours social. Regards interdisciplinaires sur une notion problématique $~{ }^{7}$; là encore, dans des ouvrages qui s'interrogent, à partir d'une 
discipline, sur les interconnexions avec d'autres disciplines ${ }^{8}$. Mais en même temps le doute s'installe sur la définition de cette notion lorsque le terme d'interdisciplinarité apparait en équivalence (parfois en complément) avec d'autres termes, comme en témoignent certains titres de colloques: «Langue/Langage et Culture. Approches interdisciplinaires et interparadigmales $»^{9} ;$ « Le Moi et l'Autre. Étude pluridisciplinaire de la différence $»^{10}$; à quoi on peut ajouter la concurrence avec d'autres termes comme multidisciplinarité et transdisciplinarité. Dans son ouvrage sur l'interdisciplinarité en analyse de discours, Frédéric Darbellay (2005) consacre ses premiers chapitres à apporter des distinctions entre ces différents termes. Avant même d'en avoir pris connaissance, j'avais moi-même proposé de distinguer inter- de pluri-disciplinarité (Charaudeau, 2007a), et remarqué la communauté de points de vue. Je n'entrerai pas dans le détail de ces distinctions, mais, comme cet auteur, je considère que la pluridisciplinarité est « une addition de disciplines, sans véritable interaction entre elles » (Darbellay, $2005: 46$ ), ce qui fait que chaque discipline garde son autonomie, ne réinterroge pas ses présupposés au regard de l'autre discipline, et ne fait qu'apporter (entreprise salutaire) son propre éclairage sur un objet d'étude lui-même analysé par d'autres. Il s'agit d'une juxtaposition de points de vue qui délivrent chacun une connaissance particulière sur le phénomène étudié. Par exemple, à l'heure actuelle, le cNRS met en place des programmes de recherche associant des spécialistes des disciplines du «vivant " (biologie humaine ou animale, biodiversité, évolution, écologie) à des spécialistes des sciences humaines et sociales (sciences du langage, sciences de l'information et de la communication, sociologie). Les premiers, préoccupés par la façon dont les résultats de leurs travaux circulent dans l'espace public, cherchent à savoir comment les discours tenus par les scientifiques sont traités, transformés, voire dévoyés, par les médias. Si ces scientifiques s'intéressent à ce que peut leur apporter d'autres disciplines, c'est de façon périphérique, dans la mesure où cela n'est pas destiné à remettre en cause leur propre discours en tant que scientifiques. Il s'agit ici d'un appel à collaboration d'autres compétences pour traiter d'une question qui dépasse le champ de compétence initiale. On pourra également citer le cas de colloques qui convoquent plusieurs disciplines pour que chacune apporte son point de vue sur un même phénomène touchant la santé, la sécurité, l'environnement, etc. Dans ce cas, on parlera de multidisciplinarité que l'on distinguera de la pluridisciplinarité en ce que cette dernière est centrée sur un questionnement commun aux disciplines concernées, alors que la première est ouverte, sans limites, sur de grandes questions, sans problématisation particulière ${ }^{11}$. La transdisciplinarité, comme invite à penser le préfixe trans-, correspond à un mouvement de traversée des disciplines aboutissant à une « coconstruction des savoirs qui traversent littéralement les disciplines constituées " (Darbellay, 2005 : 51). C'est, me semble-t-il, la démarche que suivent, bien que de façons différentes, Roland Barthes (entre littérature, philosophie, psychanalyse), Michel Foucault (entre philosophie et histoire) et Edgar Morin (entre les différentes disciplines de la connaissance). Il s'agit d'une intégration des savoirs de diverses disciplines de telle sorte qu'émerge un discours sui generis construisant son propre lieu de pensée ${ }^{12}$. L'interdisciplinarité est plus difficile à réaliser, elle «n'est pas de tout repos » comme le dit Roland Barthes (1984: 69), car elle consiste à établir de véritables connexions entre concepts, outils d'analyse et modes d'interprétation de différentes disciplines. Il ne suffit pas d'ajouter des disciplines sur un même objet d'analyse; il faut faire se confronter diverses compétences disciplinaires afin de rendre plus pertinents ces concepts et outils d'analyse, ou d'étendre le champ des interprétations à partir de résultats eux-mêmes issus de protocoles d'analyse communs. C'est ce que je voudrais envisager. Mais 
auparavant, il convient de s'interroger sur ce qui est commun aux sciences humaines et sociales.

\section{Le champ disciplinaire}

3 Une discipline est constituée d'un certain nombre de principes fondateurs, d'hypothèses générales, de concepts qui déterminent un champ d'étude et permettent en même temps de construire le phénomène en objet d'analyse. Se constitue ainsi un cadre conceptuel, et c'est à l'intérieur de celui-ci que peuvent être construites diverses théories, comme proposition d'une systémique autour de certaines catégories. Sans cadre théorique, point de discussion possible, car on ne saurait dire au nom de quoi on pourrait évaluer, confirmer ou contester les résultats d'une analyse. Il s'agit là d'un principe de pertinence : discuter les explications que l'on donne sur le monde n'est possible que si l'on connait le cadre conceptuel de référence, les catégories, les modes de raisonnement et les procédures d'analyse dont elles dépendent. Pour être opérationnel, ce cadre conceptuel a besoin de se doter d'outils de description et de probation qui permettent à la fois de construire l'objet d'analyse, de le décomposer autant que de besoin, d'effectuer des distinctions ou des rapprochements, de mettre au jour des mécanismes de fonctionnement du phénomène étudié, et de présenter le tout sous forme de résultats à interpréter. Cet outillage constitue une méthodologie, et c'est ce couple théorieméthodologie qui fonde une discipline en en déterminant le lieu de pertinence. Cependant, il peut se faire que plusieurs positions théorico-méthodologiques coexistent à l'intérieur d'un même cadre conceptuel. Mais dans la mesure où ces positions partagent les propositions fondatrices du cadre, on dira qu'elles appartiennent à un même champ disciplinaire. C'est ce qui donne lieu à l'existence en son sein de sous-disciplines ${ }^{13}$. Ainsi en est-il du champ des sciences psychologiques qui se subdivise en sous-disciplines (psychologie cognitive, psychologie sociale, psychologie clinique) mais qui se réfèrent toutes à une certaine conception du psychologique par opposition, par exemple, au sociologique ou à l'anthropologique. Ainsi en est-il du champ des sciences du langage qui se subdivise en diverses sous-disciplines (linguistique descriptive de la langue, linguistique cognitive, sociolinguistique, ethnolinguistique, linguistique du discours) mais qui se réfèrent toutes à des concepts communs de signe, forme et sens, ordres syntagmatique et paradigmatique, énoncé/énonciation et de procédures de recueil et analyse de corpus. Il en est de même pour la sociologie, l'anthropologie et l'histoire. Cette notion de champ disciplinaire est utile pour éviter certaines querelles qui ont lieu au sein des sciences humaines et sociales déniant aux sciences de l'éducation et aux sciences de l'information et de la communication le label de discipline. On a affaire à deux champs disciplinaires qui circonscrivent, chacun a sa façon, leur domaine d'étude, et qui, selon l'objet qu'ils étudient, peuvent avoir recours, autant que de besoin, à d'autres disciplines, telles la sociologie, la psychologie sociale, les linguistiques de la langue et du discours, l'histoire, etc. On peut même constater que, parfois, s'instaurent, à l'intérieur de chacune de ces sous-disciplines, des courants disciplinaires proposant des hypothèses, des catégories et une démarche d'analyse spécifiques. C'est le cas en sociologie avec les courants interactionniste, compréhensif, critique, dans le droit fil d'un constructivisme social (voir Corcuff, 1995). C'est également le cas en analyse du discours, sous-discipline appartenant au champ des sciences du langage, à l'intérieur de laquelle on constate divers courants, les uns plutôt historicisants, d'autres idéologisants, d'autres plus formels, 
d'autres davantage communicationnels ${ }^{14}$. À l'évidence, cela a produit un éclatement disciplinaire du champ des sciences humaines et sociales, auquel se sont parfois ajoutés techniques d'analyse nouvelles ou positionnements idéologiques faisant parfois École, ce qui fait que toute classification entre champ disciplinaire, discipline, sous-discipline et courant peut prêter à discussion. Mais mon propos n'est pas ici de procéder à une telle classification (voir Boure, 2007). Il est de rappeler qu'à l'intérieur des sciences humaines et sociales, il existe des disciplines, que celles-ci se fondent sur des présupposés théoriques, qu'elles utilisent une méthodologie et qu'elles donnent lieu à diverses pratiques analysantes, car faute d'un tel cadre, toute explication n'est que commentaire et non point analyse - sur le monde. Le commentaire se refuse à catégoriser, il globalise et procède par affirmations essentialisantes, se donnant comme une explication naturelle; l'analyse, elle, catégorise et procède à des distinctions selon certains critères pour ensuite montrer les combinaisons possibles, proposant des phénomènes une explication culturelle, certes relative, mais pouvant être discutée et ayant une fonction critique.

\section{Caractérisation des phénomènes sociaux et problématisations}

4 Les phénomène sociaux peuvent être considérés comme des ensembles de signes ( $\mathrm{y}$ compris d'action) qui relèvent à la fois de raisons structurelles, dans la mesure où ils s'organisent selon des normes récurrentes et reproductibles, plus ou moins stables, plus ou moins institutionnalisées, lesquelles surdéterminent en partie les actions sociales; de raisons processuelles, dans la mesure où ils résultent d'actions stratégiques mises en place par des sujets qui cherchent à s'individuer au regard des contraintes structurelles, stratégies qui finissent par faire évoluer ces structures. Définis sous ce double aspect structurel et processuel, les phénomènes sociaux témoignent des différentes pratiques sociales qui s'instaurent dans une société. Si l'on observe les pratiques analysantes qui s'attachent à décrire ces phénomènes, on peut constater qu'elles se distribuent autour de trois grandes problématisations.

Une qui s'intéresse à repérer et décrire les processus de régulation sociale : les individus et les groupes sociaux, soumis à des rapports de force dans lesquels ils cherchent à constituer leur identité face à celle de l'autre (principe d'altérité) par un jeu d'attraction et de rejet, établissent à force d'échanges (de comportements et de paroles), et par récurrence, des liens qui leur permettent de se reconnaître. C'est à décrire ces comportements, les rôles sociaux et les identités qui les caractérisent, que s'emploient différentes disciplines, chacune à sa façon. On peut considérer que c'est au terme de ces processus de régulation que se constituent des normes d'usage : les normes langagières qu'étudient la sociolinguistique de façon corrélationnaliste, l'ethnométhodologie et l'analyse du discours lorsqu'elle s'emploie à déterminer des genres discursifs; les normes des cadres d'expérience qu'étudie la sociologie interactionniste; les normes relationnelles qui structurent les sociétés qu'étudie l'anthropologie autour de l'hypothèse $\mathrm{du}$ «don» comme enchaînement logique d'intérêts entre des sujets donneurs et receveurs qui sont impliqués de façon consubstantielle autour d'un objet sacralisé (Godelier, 1982 ; 1997) ; les normes organisationnelles des processus de communication à travers la description des divers dispositifs qui les mettent en scène auxquelles 
s'intéressent les sciences de l'information et de la communication et la médiologie (Debray, 1994).

6 Une problématisation relative à la question des rapports de force qui s'instaurent entre les individus et les groupes sociaux, à l'intérieur même du jeu de régulation sociale, ce qui conduit les sujets à jouer de stratégies pour s'imposer à l'autre (rapport de domination), sortir du rapport de domination (contre-pouvoir), trouver des alliances (jeu de persuasion), se faire aimer de l'autre (jeu de séduction). Ce principe d'influence engendre les notions de stratégies, d'influence et, de nouveau, d'identité psycho-sociale, notions qui sont au centre de diverses disciplines: la psychologie sociale qui étudie, de façon expérimentale, les processus d'influence; la sociologie s'intéressant à décrire les phénomène de domination ; l'analyse du discours qui décrit les stratégies discursives des sujets d'énonciation qui sont mises à l'œuvre dans divers types de discours (publicitaire, politique, médiatique).

7 Une problématisation s'intéressant à la façon dont se construisent et s'organisent les systèmes de pensée en savoirs, savoirs de connaissance et savoirs de croyance (Charaudeau, 2004). Cette problématisation fait émerger les notions de représentations sociales, d'imaginaires sociaux et d'idéologies qui, elles aussi, sont au centre de diverses disciplines, même si chacune d'entre elles en propose une définition qui lui est propre : l'anthropologie sociale comme archétypes à travers l'étude des mythes et récits; la sociologie comme imaginaires collectifs à travers l'observation des comportements, de l'organisation des institutions et des enquêtes de terrain; la psychologie sociale comme processus de compréhension guidant les conduites; l'analyse de discours cherchant à révéler les idéologies sous-jacentes aux discours des différents acteurs sociaux.

Cette vue d'ensemble sur les problématisations d'étude des phénomènes sociaux permet de voir comment, à partir de ces notions communes, les disciplines des sciences humaines et sociales construisent leurs objets selon leurs présupposés théoriques et leur instrumentation méthodologique : certaines privilégient des études de terrain avec des procédures d'observation et d'enquête (sociologie et l'anthropologie), d'autres des procédures d'expérimentation (psychologie sociale), d'autres des analyses de corpus avec des procédures plus ou moins systémiques de recueil et traitement du matériel sémiologique réuni (histoire, sciences du langage). Mais on voit en même temps ce que ces diverses disciplines ont en partage: sociologie, psychologie sociale, anthropologie sociale, sciences du discours et de la communication - pour ne prendre que celles-ci - ont en commun non seulement des notions mais aussi des problématisations, c'est-à-dire un même cadre de questionnement. Ce qui les conduit à avoir recours, autant que de besoin, aux autres démarches et procédures. Certes, études de terrain, études expérimentales et études de corpus réfèrent à des cadres théorico-méthodologiques différents, mais elles peuvent se compléter ou même interférer selon les questionnements qui surgissent au cours d'une recherche. C'est que les sciences humaines et sociales mettent en œuvre une double démarche: "empirico-inductive» qui va de l'observation des phénomènes sociaux, selon certaines méthodes de recueil des données, à la description d'un objet construit en catégories interprétatives en fonction d'un certain outillage méthodologique (procédure up) ; " hypothético-déductive ", plus conceptuelle, qui va d'hypothèses et de catégories préalablement établies à la vérification de la validité de l'instrumentation utilisée. C'est dans un mouvement de va-et-vient entre ces deux démarches, discutées depuis Aristote en passant pas Kant et la phénoménologie quant à la façon de tenir à la fois ce qui relève des sens et ce qui relève de la raison, que s'élaborent concepts et 
instruments d'analyse, et que sont mises en œuvre les interprétations. Toute discipline des sciences humaines et sociales s'affronte au problème du repérage des données pertinentes, de leur recueil, de leur classement selon certaines catégories, de leur analyse et de l'interprétation des résultats. De ce point de vue, on peut dire que cette double démarche est d'ordre constructiviste ${ }^{15}$. Ainsi comprend-on qu'un même phénomène ou domaine de pratique sociale puisse être construit en divers objets d'analyse, tout en dépendant d'une même problématisation. Par exemple, les domaines de pratique politique, médiatique, juridique ou éducatif seront construits en objet d'études sociologique, psychosociologique, anthropologique ou discursif, mais en même temps, ces objets seront traversés par des notions communes (identités, représentations, influence et persuasion). Il n'y a donc pas, en la matière, de domaine d'étude réservé. Par exemple, l'étude des phénomènes du politique n'est pas, comme certains voudraient le laisser croire, l'exclusivité des sciences politiques, pas plus que de la sociologie politique, de l'analyse des discours politiques ni même de la philosophie politique. Chaque approche disciplinaire opère une ponction dans chacun de ces domaines créant ainsi un lieu de pertinence d'analyse qui lui est propre. La question demeure cependant celle de leur articulation possible.

\section{Pour une interdisciplinarité « focalisée »}

9 L'interdisciplinarité, c'est l'effort d'articuler entre eux les concepts, les outils et les résultats d'analyse de différentes disciplines. Cela ne peut se faire avec plusieurs disciplines à la fois, car, pour pouvoir procéder à une interrogation ou un empreint entre des concepts, il faut pouvoir les considérer dans leur cadre théorique afin de ne pas les déformer, les interroger à la lumière d'une autre discipline et expliquer dans quelle mesure et à quelles fins d'analyse ils peuvent être empruntés et intégrés dans l'autre discipline. Ce travail ne peut être fait qu'à travers la mise en regard de deux disciplines, quitte à ce que cette opération soit démultipliée, c'est-à-dire répétée de discipline à discipline. On proposera quelques exemples d'articulation autour de notions, d'outils et du recours à d'autres disciplines à des fins d'interprétation.

\section{Des notions en question}

10 La notion d'identité est une de ces notions qui, depuis un certain temps, fait l'objet de réflexions interdisciplinaires. Elle est centrale en sociologie, en psychologie sociale, en anthropologie, en sémiologie, en analyse du discours et chacune de ces disciplines la définit d'une façon qui lui est propre. Cette notion est liée à la question du sujet qui est elle-même définie différemment selon ces disciplines, et selon les époques. On sait que, depuis la fin des années 80 , on a parlé, dans différentes disciplines des sciences humaines et sociales, d'un «retour du sujet" signifiant par là une rupture avec le modèle structuraliste qui l'avait nié dans son altérité et l'avait essentialisé comme porteur de social et d'idéologie. Plus exactement, il était bien question de sujet, mais ce sujet était la société ou le social ou le pensé social, bref une entité abstraite, un système qui, à travers ses diverses configurations étatiques et institutionnelles, surdétermine les acteurs. Ainsi était-il possible de prétendre mettre en évidence (voire dénoncer) les rapports de domination qui s'instaurent dans la société, à travers un processus d'objectivation, et en évitant d'entrer dans la subjectivité des acteurs sociaux considérée comme dangereuse 
pour l'établissement de la vérité. Le sujet revient donc en force, bien que de façon différente, chez certains sociologues comme Alain Touraine (2007: 32) qui, reprenant l'idée de "souci de soi » que Michel Foucault développait dans se derniers cours au Collège de France, propose de distinguer le moi personnel de l'individu et celui de l'acteur social pour redéfinir le sujet dans une perspective d'altérité «le plus haut niveau de formation du sujet [étant] celui de la reconnaissance de l'autre comme sujet ». On le voit aussi chez Michel Wieworka (2007) pour qui le sujet est «ce qui rend capable d'être acteur, si les conditions le rende possible, il est ce qui permet d'agir, de construire son expérience, de la maîtriser, du moins jusqu'à un certain point» (ibid.: 16); et chez Bernard Lahire (2005) qui étudie comment l'acteur incorpore des "schèmes d'action". Les philosophes ne sont pas en reste. Les uns, comme Norbert Elias (1991) qui, dans une filiation phénoménologique, cherche à éviter l'opposition entre «individus » et "société" et propose une sociologie dont l'objet est constitué d'individus interdépendants; d'autres comme Paul Ricœur (1990) qui, dans une perspective herméneutique, trouve un sujet double. Mais aussi les historiens qui, sous la poussée de l'école des Annales, réintroduisent dans leur champ d'analyse les individus en tant qu'ils sont acteurs et responsables des événements à travers leurs comportements et les représentations normées qu'ils construisent (consciemment ou non), lesquelles expliquent en retour les événements. On pourrait également l'observer en anthropologie et en psychologie sociale. Mais c'est son histoire dans les sciences du langage qui est particulièrement éclairante pour comprendre les influences qui se sont produites entre disciplines. Les sciences du langage ont commencé par définir un sujet ayant une identité d'être potentiellement parlant, porteur des possibilités qu'offre le système de la langue, et une identité d'être effectivement parlant réalisant son acte de langage. Cette opposition a été différemment dénommée selon les modèles qui en rendaient compte, chacun apportant des spécifications propres. Chez Ferdinand de Saussure, l'opposition langue/parole justifiant l'évolution des structures de la langue par l'effet de retour de l'exercice individuel de la parole ; le sujet est ici une entité dite sociale mais, en réalité, disparaissant dans le système de la langue. Chez Noam Chomsky, c'est l'opposition compétence/performance qui, s'inscrivant en contre du "fixisme» structuraliste, définissait un sujet « locuteur-auditeur idéal » se livrant à des opérations mécaniques de construction de phrases (en performance) à partir de structures profondes, à l'aide de règles de transformation (en compétence) permettant de produire des énoncés : le sujet compétent n'est ici qu'un acteur abstrait d'opérations dont on ne sait si elles sont d'ordre cognitif ou purement machinique. Ainsi peut-on dire que le sujet était absent du structuralisme saussurien et du générativisme chomskyen, mais il le fut également de l'analyse du discours, d'inspiration althussérienne, initiée par Michel Pêcheux (1990). Le sujet n'est plus celui de la langue, mais du discours en tant qu'il est porteur d'idéologie ${ }^{16}$. Ce n'est donc pas lui qui parle mais la société à travers lui : le sujet n'agit pas, il est agi, de même, le sujet ne parle pas, il est parlé. On ne peut donc le saisir puisque ce sont les discours, les « formations discursives » dira Michel Foucault, qui circulent dans la société et qui imposent des rapports de force dont le sujet est en quelque sorte l'otage. Est ensuite apparue l'analyse pragmatique du langage de source anglo-saxonne (John L. Austin, John Searle), ce qui a eu le mérite de sortir les études sur le langage d'une immanence qui les empêchait de prendre en compte les forces des actes de langage susceptibles de produire des effets sur leurs destinataires. Ainsi apparaît un premier degré d'altérité par la prise en compte d'un sujet destinataire plaçant du même coup le sujet locuteur dans un rapport d'influence à celui-ci, les deux liés par un postulat 
d'intentionnalité. Parallèlement, et en partie sous l'influence de la pragmatique, s'opère en France un retour sur Émile Benveniste mettant «l'homme au centre du langage » et définissant le sujet dans un rapport d'«intersubjectivité » $J e-T u$, à travers un acte d'énonciation. Cela déclenche nombre de travaux sur l'énonciation et Oswald Ducrot développe une théorie polyphonique du langage dans laquelle il propose de cliver le sujet en locuteur et énonciateur, montrant par là même que tout énoncé est porteur de plusieurs voix. Les théories de la pragmatique et de l'énonciation ont constitué un premier tournant dans les analyses linguistiques en ce qu'elles ont ouvert une porte, comme le dit Dominique Maingueneau (2002) à l'interrogation sur la dimension psychologique et sociale du sujet ${ }^{17}$. Mais en ce qui concerne l'identité, les choses ne sont pas encore jouées, car le sujet reste un sujet opérateur de langage sans que soient véritablement prises en compte les conditions situationnelles de production de l'acte de langage ${ }^{18}$, et donc sans que soit problématisée la question de l'identité du sujet. Ce n'est que sous l'influence de l'ethnographie du langage (Dell H. Hymes, John J. Gumperz), de la sociolinguistique (Basil Bernstein, William Labov), de l'ethnométhodologie (Howard Garfinkel pour qui «les "personnes", "personnes particulières" et "individus", sont des aspects observables d'activités ordinaires ${ }^{19}$ ), de l'interactionnisme verbal et de l'analyse des conversations (Harvey Sacks, Emmanuel A. Schegloff, Gail Jefferson), reprise et développée en France par Catherine Kerbrat-Orechionni particulièrement pour l'étude de la politesse, et de la sociologie du langage (Erving Goffman) qu'a commencé à apparaître la prise en compte de l'identité sociale et psychologique du sujet parlant en relation avec celle de son interlocuteur. Cette nouvelle orientation a été confortée, d'un côté par les travaux des psychosociologues du langage (Claude Chabrol, Alain Trognon, Rodolphe Ghiglione, Jean-Léon Beauvois) qui s'employaient à intégrer dans leur champ des notions de pragmatique et d'énonciation, parfois éprouvées expérimentalement, de l'autre, par les études de communication sur les médias s'interrogeant à la fois sur les processus de production du discours selon le statut des acteurs et de leur réception en fonction des catégorie de récepteurs. Il en est résulté des influences réciproques quant au traitement de la question de l'identité du sujet parlant dans chacune de ces disciplines, même lorsque les emprunts ne sont pas explicites. Pour ce qui me concerne, je travaille depuis longtemps avec cette problématique de dédoublement du sujet, sujet communiquant ayant une identité sociale et sujet énonciateur ayant une identité discursive, dédoublement qui permet de rendre compte des contrats et stratégies des discours médiatiques et politiques (Charaudeau, 2009). Si, dans le champ des études de discours, se pose la question du rapport entre identité sociale et identité discursive du sujet parlant, c'est du fait de cette mise en regard entre ces courants théoriques introduisant l'idée d'un sujet à fonctions multiples se construisant des identités tantôt plus particulièrement langagières (comme dans les théories de l'énonciation, de la pragmatique et des genres), tantôt plus communicationnelles en rapport avec des identités sociales et psychologiques pour analyser les jeux de persuasion sociale ${ }^{20}$. La notion de représentation associée à celle d'imaginaire est également une de ces notions qui se trouvent au centre de plusieurs disciplines, particulièrement en sociologie, psychologie sociale et anthropologie. Elle apparaît chez Émile Durkheim sous la dénomination de "représentations collectives ", dénomination contestée par Serge Moscovici parce que le terme de collectif renvoie davantage à un groupe fermé sur lui-même et à des opinions collectives intracommunautaires. Celui-ci propose alors l'expression "représentations sociales », plus générique, qui inclut les représentations collectives sans préjuger de son degré de généralisation, parce qu'il s'agit de définir une notion qui explique et justifie, les 
pratiques sociales, leurs normes et leurs règles. L'individu ne pouvant se contenter d'agir, il lui faut se donner une raison d'agir, des motifs et des finalités qui lui permettent de porter des jugements sur le bien fondé de ses actions; il doit donc se les représenter en interaction avec les autres, et, en se les représentant, il fait exister et invente la société qui l'invente dans le même temps (Rouquette, Rateau, 1998). Par voie de conséquence, les représentations sociales sont un mode de connaissance du monde socialement partagé. L'imaginaire est une notion qui a sa propre histoire. Dans la pensée classique, l'imagination était considérée comme fantasia ; elle était du côté de la folie ( «la folle du logis ») qui s'opposait alors à la raison, seule capable de gérer le face à face entre l'Homme et le Monde. Pensée classique qui se poursuit jusqu'au XVIII ${ }^{e}$ siècle. En affirmant l'existence d'une double conscience chez l'homme, double conscience qui se croise avec la dualité d'un "soi individuel » et d'un "soi collectif », Sigmund Freud place l'imaginaire dans la seconde topique (" Ça / Moi / Surmoi »), du côté du "Surmoi », le « Ça » étant de l'ordre du Symbolique. Sur la lancée, Carl G. Jung développe l'idée d'«archétypes » comme ensemble de thèmes récurrents construisant des imaginaires personnels reposant sur un fond commun d'inconscient collectif. Parallèlement, Gaston Bachelard (ils sont contemporains à un an près) oppose la " conceptualisation ", activité rationnalisante qui produit la science, et la "rêverie », activité créatrice produisant une vision poétique du monde ; mais ces deux activités sont liées en ce qu'elles sont à l'origine des principes organisateurs des conduites humaines. Toujours parallèlement, l'anthropologie considère les rituels sociaux, les mythes et les légendes comme des discours qui témoignent de l'organisation des sociétés humaines. C'est semble-t-il dans la ligne de ces définitions que Cornelius Castoriadis (1975) introduit la notion d'imaginaire social qu'il applique aux cas où cette activité représentationnelle du monde se fait dans un domaine de pratique sociale déterminé (artistique, politique, juridique, religieux, éducatif, etc.), afin de rendre cohérent le rapport entre l'ordre social et les conduites, et de cimenter le lien social à l'aide des appareils de régulation que sont les institutions. Ajoutons que l'imaginaire social est à dimension variable, du fait de la plus ou moins grande extension du groupe, du jeu des comparaisons possibles entre groupes, et de la mémoire collective du groupe qui se construit à travers l'histoire. Ainsi peut-on parler aussi bien d'imaginaires qui s'inscrivent dans la personne (perception différenciée de la mort selon qu'elle s'inscrit dans l'histoire intime de l'individu ou qu'elle touche un sentiment d'appartenance communautaire), que d'imaginaires plus proprement collectifs qui varient selon la nature du groupe. Ici n'est pas le lieu d'entrer dans le détail de ces définitions ni de les discuter, mais de constater la présence du mécanisme de représentation dans diverses disciplines. En histoire, lorsque celle-ci part en quête des mentalités. Parfois, dans la collaboration entre un anthropologue et une linguiste: Dan Sperber et Deirdre Wilson, dans leur théorie de la pertinence, parlent de "représentations partagées ", notion fondatrice de l'activité de langage qui repose sur l'idée d'adhésion des membres d'un groupe à des valeurs communes qui feraient consensus pour qu'ils puissent communiquer. En analyse du discours, sont employés les termes d'idéologie et de formation discursive par Michel Pêcheux (1990), puis par Jacques Guilhaumou (1993) et Régine Robin (1973) qui les rapportent à l'histoire dans une double filiation althussérienne et foucaldienne; de même, mais avec d'autres méthodes, par Maurice Tournier (1992:9) pour qui « les mots n'ont pas en eux-mêmes une histoire [car] c'est l'histoire qui se construit avec eux, en habitudes, dans son événement c'est-à-dire son récit », ce qui fait qu'ils sont témoins d'idéologie. On peut également citer les travaux anglo-saxons autour de la Critical Discourse Analysis (Norman Fairclough, Theo Van Leeuwen, Teun A. Van Dijk, Ruth Wodak) 
qui cherchent, à travers l'interrelation entre un contexte social et son appropriation subjective par le sujet, à décrire la façon dont « les mécanismes mentaux contrôlent les conduites langagières» (Petitclerc, Schepens, 2009: 13). Par ailleurs, Anne-Marie Houdebine (2002), dans la lignée d'André Martinet qui, dans les années 70, lance des enquêtes sur la phonologie du français contemporain pour mettre en évidence la façon dont les individus valorisent ou dévalorisent leur parler, développe la notion d'imaginaire linguistique. De son côté, la didactique des langues cherche à introduire dans les procédures d'enseignement la question des différences culturelles, ce qui la conduit à analyser les stéréotypes comme porteurs de représentations culturelles. Pour ma part enfin, j'ai tenté de me réapproprier la notion d'imaginaire en la définissant en termes d'imaginaire sociodiscursif (Charaudeau, 2007b), non pas comme un concept, mais comme un mécanisme d'engendrement discursif des savoirs de connaissance et de croyance qui façonnent la réalité en réel signifiant. Cette question est délicate à traiter. Elle relève d'une théorie de la connaissance, et l'on sait, depuis l'origine de la philosophie qu'il en est plusieurs, sans compter le grain de sel qu'apportent les sciences humaines et sociales. Ce qui semble acquis - malgré encore des oppositions qui ressemblent davantage à de la défense de territoires qu'à de la controverse scientifique - est qu'on ne peut dissocier représentation et action, et donc activité cognitive, pratique sociale et conscience agissante des sujets. Ceux-ci sont dotés de la faculté de percevoir et d'évaluer les actions collectives, ce qui leur permet d'ajuster leurs conduites en fonction de ces représentations du monde, des situations dans lesquelles ils agissent et des places qu'ils pensent qu'occupe autrui. Autrement dit, il semble acquis qu'on ne peut dissocier connaissance savante, croyances sociales et connaissance ordinaire, bien qu'il soit nécessaire de le faire pour les rendre opératoires. Ce que l'on peut dire - qui traverse ces différentes disciplines - est que les représentations et les imaginaires sont engendrés par les discours qui circulent dans les groupes sociaux (à travers des relations interdiscursives ou dialogiques), s'organisant en systèmes de pensée cohérents, créateurs de valeurs, jouant le rôle de justification de l'action sociale et se déposant dans la mémoire collective. Il y a bien d'autres notions communes à différents champs disciplinaires et qui, du fait de leur mise en regard, font avancer la recherche dans chacun d'eux. Parfois même, c'est à l'intérieur d'un même champ que se produisent des influences. Ainsi en est-il de la notion de stratégie que l'on trouve en psychologie cognitive (Esperet, 1990), en psychologie sociale (Chabrol, 1990) et en analyse des discours permettant de distinguer persuasion et influence sociale; de la notion de dispositif que l'on trouve en sciences de l'information et de la communication, reprise dans le cadre d'analyses discursives des médias et du politique, car elle joue un rôle structurant des productions langagières (Lochard, 1999); de la notion d'émotion, autrefois traitée par la rhétorique aristotélicienne, puis pratiquement réservée à la psychologie et qui a investi les études de discours en rapport avec les phénomènes conversationnels et les genres propagandistes - publicité, promotion, politique (Plantin, Doury, Traverso, 2000 ; Rinn, 2008) -, notion évidemment redéfinie dans chacun de ces cas en croisant d'autres notions comme celles d'opinion, de propagande et de manipulation.

\section{Des outils transversaux}

11 Il est une autre sorte d'interdisciplinarité, celle de l'emploi de mêmes outils d'analyse dans différentes disciplines, outils d'analyse que l'on peut qualifier de transversaux, comme l'informatique et le calcul statistique. Ces outils sont utiles et performants pour ce 
qui concerne la possibilité de traiter des masses de données avec une grande rapidité d'exécution. Du même coup, ils rendent visibles des caractéristiques qui passeraient inaperçues à l'œil de l'analyste. Cependant, il faut reconnaître qu'ils ne sont pas porteurs d'une problématisation particulière. L'utilisation de ces outils s'inscrit dans une démarche inductive cherchant à recueillir, décrire et classer les données observées dans différents objets et phénomènes, selon des critères de forme, avec un idéal d'exhaustivité. Le travail est donc ici de méthodologie techniciste pure, dans la mesure où il n'y a pas d'hypothèses particulières orientant la recherche dans une direction ou une autre. Les données, les indices de description et les catégories de classement sont pensées simultanément avec l'outil technologique le plus performant possible pour le recueil des données, leur classement et les modes de représentation afin de fournir à l'utilisateur divers modes de consultation. Il s'agit d'une démarche descriptiviste, centrée sur des archives, alors que toute discipline suit, d'une façon ou d'une autre, une démarche constructiviste $^{21}$ élaborant son propre objet, la question étant de savoir dans quelle mesure l'accumulation des données empiriques, par quelque procédé que ce soit, permet de comprendre le phénomène étudié. Cela dit, le recours à ces outils a permis d'introduire dans certaines disciplines des études quantitatives qui servent de socle à des études qualitatives par le biais d'échantillons raisonnés et maîtrisés. Ainsi voit-on apparaitre, dans les études portant sur les médias et les discours politiques, des associations heureuses entre des analyses quantitatives permettant de construire des corpus et de rendre compte des récurrences de certaines caractéristiques selon des modèles plus ou moins sophistiqués (Tournier, 1992), des analyses sémantiques, des analyses argumentatives, en relation avec la prise en compte de données communicationnelles dispositifs, identités des locuteurs, genres discursifs, types d'échange, etc. (Charaudeau, 2001).

\section{Le recours nécessaire à d'autres disciplines pour l'interprétation}

L'interdisciplinarité peut également s'exercer d'une autre façon: en mettant en regard différentes disciplines sur un même objet. Cela pourrait renvoyer à ce que j'ai défini comme étant de la pluridisciplinarité, distinguée de l'interdisciplinarité. Mais il s'agit ici, soit de préparer le travail d'une discipline par des analyses réalisées dans une autre à propos d'un même objet d'étude, soit de prolonger les analyses fournies dans le cadre d'une discipline par le recours à une autre. Par exemple, pour l'étude d'un objet comme l'histoire des habitudes alimentaires en France, on voit que les historiens sont amenés à collaborer avec des géographes, des anthropologues et même des urbanistes. Chacune de ces disciplines apporte ses propres résultats en utilisant ses outils d'analyse, mais cela se fait dans une collaboration étroite, tentant d'articuler entre eux les résultats d'analyses de corpus, d'études de terrain ou d'expérimentations, pour aboutir à une vue d'ensemble du phénomène étudié. Cette pluridisciplinarité, à la différence de celle que j’ai définie comme une simple addition de disciplines - chacune livrant ses propres résultats - est une pluridisciplinarité articulée et intégrée autour d'un protocole d'analyse commun. Je préfère l'appeler interdisciplinarité. On la voit mise en œuvre dans la rubrique «Échanges » de la revue Questions de communication sous la forme de discussions critiques permanentes sur «des concepts ou des méthodes utilisés dans les travaux sur l'information et la communication» (cité dans la 2e page de couverture de la revue). Celle-ci peut aussi se réaliser dans un mouvement de prolongement d'une analyse par une autre qui apporte un éclairage complémentaire du fait d'études de terrain ou de 
démarches expérimentales. Par exemple, l'analyse discursive d'une controverse mise en scène par les médias (radio, presse et télévision) qui serait prolongée par une étude de terrain menée par des sociologues pour en vérifier la réception, mais en tenant compte des résultats de l'analyse précédente. C'est ce que le Centre d'analyse du discours (CAD) de l'université Paris 13 s'est employé à développer lors de ses travaux sur des objets médiatiques en collaboration avec des psychosociologues ${ }^{22}$. De même, il est difficile d'étudier certaines questions comme la « propagande » sans faire étroitement collaborer l'histoire, l'anthropologie, la sémiologie, les sciences de l'information et la science politique.

\section{Le problème de l'interprétation}

Il est un moment de l'étude d'un phénomène que tout chercheur aborde avec une certaine appréhension, celui de l'interprétation des résultats de ses analyses, où il s'agit de rendre le monde un peu plus intelligible. Car si chaque discipline, à l'aide ses présupposés théoriques et de son cadre méthodologique, permet au terme d'une analyse d'établir des résultats, encore faut-il que ceux-ci soient interprétés. Or, interpréter n'est pas une mince affaire, d'autant qu'il y a plusieurs façons de concevoir l'interprétation, les unes plutôt finalistes, les autres davantage causales. Il faudrait se lancer dans un long développement critique pour discuter les différences entre des notions telles que compréhension, explication et interprétation. Ce n'est pas ici le lieu et je renvoie, entre autres éléments, à l'exposé qu'en fait Paul Ricœur (1990a). Il discute les notions d'explication et de compréhension, à partir des positions de Wilhem Dilthey sur les sciences de l'esprit (1883) sur la naissance de l'herméneutique, et de celles des philosophes du langage qui, à la suite de Ludwig Wittgenstein, distinguent causalité et motivation. Il tient à justifier l'opposition de ces deux notions à l'inverse de Wilhem Dilthey qui ne retient que la seule compréhension, et à l'inverse du Cercle de Vienne qui donne la primauté à l'explication. Car, pour lui, il n'y aurait pas deux champs scientifiques (les sciences de la nature d'un côté, les sciences de l'esprit de l'autre), mais une "unité de la science " qui repose sur les mêmes procédures explicatives. Aussi propose-t-il de maintenir le lien entre expliquer et comprendre en donnant la primauté au comprendre et en considérant l'explication comme une médiation nécessaire et cependant secondaire, et en subordonnant le comprendre à l'interpréter. Mais l'approche herméneutique est elle-même discutée, et l'on pourra se reporter à certains travaux qui en font état ${ }^{23}$, ainsi qu'au point de vue de Jürgen Habermas dans sa Théorie de l'agir communicationnel (1981) dont l'orientation combine psychologie sociale et descriptions structurelles. Je ne retiendrai que ce qui permet de soutenir l'idée d'interdisciplinarité que je défends, et proposerai provisoirement, sous la seule notion d'interprétation, car il s'agit ici de l'interprétation du sujet analysant, et non du sujet récepteur (lecteur, auditeur, interlocuteur, spectateur) pour lequel je préfère réserver la notion de compréhension, mouvement de saisie du sens qu'il construit (comme lorsqu'on dit : «j'ai compris» ou «je te comprends»), selon le principe de réflexivité que propose l'ethnométhodologie, qui veut que les sujets intègrent dans leur faire social (langagier ou actionnel) les motifs et représentations qui le justifient. Une distinction entre deux démarches qui recoupent en partie certaines des définitions de Paul Ricœur. Interpréter, c'est essayer de rendre compte des résultats d'une analyse en procédant à une série de mises en relation de ces résultats, les uns avec les autres ou avec autre chose qu'euxmêmes. On peut procéder à deux sortes de mise en relation et donc à deux types 
d'interprétation. Une, interne, qui consiste à montrer comment (et non pourquoi) fonctionne le phénomène étudié par une mise en relation des différentes parties qui composent les résultats d'une analyse. Soit de façon causale comme le fait par exemple l'histoire lorsqu'elle relie des événements dans une chaîne de causalité (causes physiques), ou qu'elle propose des hypothèses sur des réseaux d'intentionnalité qui auraient présidé aux événements (causes motivantes). Soit de façon corrélative, comme lorsqu'après l'analyse d'un corpus de textes médiatiques on met en relation la description des événements, les commentaires qui en sont faits, les arguments qui s'échangent dans une polémique avec les types de locuteurs et de thèmes traités (Charaudeau, 2008a). Il est une autre sorte d'interprétation interne qui consiste à mettre les éléments des résultats en relation avec les catégories et les hypothèses à partir desquelles ont été réalisées les analyses. Il s'agit d'une mise à l'épreuve ou de vérification de la validité des concepts, des catégories et des outils qui ont présidé à cette analyse. On donnera en exemple une thèse qui avait pour but de montrer dans quelle mesure la théorie des actes de langage pouvait servir à analyser un corpus de conversations, en quoi ces catégories pouvaient être reconnues valides et quelles en étaient les limites. Il s'agit alors d'une procédure de validation montrant le pouvoir explicatif, soit de catégories, soit d'hypothèses expérimentales, soit de démarches d'analyse (génétiques, structurales, immanentistes). C'est pourquoi on peut dire que ces modes d'interprétation interne s'apparentent à une procédure d'explication ayant un certain caractère d'objectivité du fait qu'elle correspond à un mouvement hypothético-déductif avec retour de vérification.

Le deuxième type d'interprétation sera dit externe dans la mesure où les résultats sont confrontés à ceux d'autres disciplines ayant étudié un objet similaire. Dès lors, on peut confronter l'analyse des stratégies discursives opérées sur un corpus de discours politiques avec certaines hypothèses que proposent la psychologie sociale sur les procédés d'influence ou la sociologie sur les types de public concernés, ou encore la philosophie politique sur les principes et les valeurs qui fondent les régimes politiques. On sait l'influence que la philosophie a pu avoir sur l'évolution de certaines disciplines: sur la sociologie ${ }^{24}$ (Ludwig Wittgenstein et Pierre Bourdieu), sur les sciences du langage (John L. Austin, John R. Searle, Jacques Derrida), la sémiotique narrative (Paul Ricœur), et l'analyse du discours (Louis Althusser, Michel Foucault). Mais je pense ici davantage à la nécessité d'avoir recours à une démarche dialogique (intertextuelle ou interdiscursive) pour interpréter les résultats de certaines analyses de discours. Par exemple, lorsqu'on analyse un corpus de textes politiques, on ne peut se passer d'un recours à des écrits de philosophie politique dans la mesure où sous ces discours courent des références à des régimes politiques (république) des modes de gouvernance (démocratie), des théories sur l'organisation de la vie des sociétés (libéralisme, marxisme, national socialisme, fascisme), des stratégies de persuasion (populisme, propagande). Ou quand on analyse la façon dont les médias rendent compte de conflits armés ou de controverses sociales, la nécessité d'aller chercher dans d'autres écrits les imaginaires qui circulent s'agissant de la guerre, de la bioéthique, de la laïcité, etc. Le mouvement est ici de mise en regard, de corrélation, ni descendant ni montant, et dont la pertinence est dépendante des choix du sujet analysant, en quoi ce travail interprétatif et d'ordre subjectif. Mais il est la condition de production d'une interprétation qui consiste, selon le principe herméneutique, à mettre en évidence ce qui est caché, ce qui est invisible à l'observation empirique. Là est la fonction critique de toute analyse des sciences humaines et sociales, fonction non réservée à telle ou telle théorie. 

complémentaires, mais il faut avoir conscience que quand, dans l'interprétation interne, on cherche à valider le cadre théorico-méthodologique, on ne dit pas grand chose sur l'objet analysé en tant que phénomène social. En revanche, lorsque, dans un effort d'interprétation externe, on cherche à expliquer le phénomène social étudié en confrontant différents points de vue, on n'interroge pas la validité de l'analyse ellemême. On voit donc qu'une pratique analysante peut avoir pour objectif, soit de valider un cadre conceptuel avec des présupposés théoriques et une instrumentation méthodologique afin d'en délimiter la pertinence et d'en montrer l'efficacité (démarche ascendante), soit de rendre compte des phénomènes sociaux en confrontant chaque analyse à d'autres lieux de pertinence sans nier le sien propre (démarche horizontale).

Voilà quelques exemples de ce que serait une interdisciplinarité distincte d'une pluridisciplinarité. Une fois de plus, impliquer des chercheurs de disciplines différentes dans un même programme de recherche n'est pas une garantie d'interdisciplinarité s'il s'agit simplement de voir comment plusieurs disciplines traitent d'une même question sans que cela affecte en quoi que ce soit chacune d'elles. Rassembler sur un même campus des chercheurs de disciplines différentes de sorte que « les sciences humaines et sociales elles-mêmes construisent leurs propres objets et leurs méthodes dans une interaction raisonnée avec les autres sciences, dans les domaines - par exemple de l'environnement et du développement durable, de la santé ou de la communication $»^{25}$ est louable et prometteur mais n'est pas, comme annoncé dans la Charte, un gage d'interdisciplinarité. Prendre pour objet d'analyse le discours d'une autre discipline - philosophique, littéraire, historique, scientifique, didactique ${ }^{26}$ - n'est pas de l'interdisciplinarité; c'est faire de l'analyse du discours à propos d'une autre discipline, et non pas en interaction avec celleci. Toutes ces démarches, aussi nécessaires et intéressantes soient-elles, relèvent de la pluridisciplinarité (avec des formes différentes) et non point de l'interdisciplinarité. En revanche, le fait que certains courants des sciences du langage intègrent des données sociologiques, socio-communicatives ou socio-psychologiques dans leur modèle théorique ou dans leur méthodologie; le fait que des psychosociologues du langage intègrent, les uns des éléments de pragmatique (Trognon, Ghiglione, 1993), les autres d'énonciation (Chabrol, 2009); que des chercheurs des sciences de l'information et de la communication s'interrogent sur leur discipline en s'appuyant sur les travaux de sociologues, de sémioticiens et d'analystes des discours (Miège, 2000) à propos de l'analyse des médias, ou que des sociologues se situent à un carrefour entre la science économique et la philosophie politique ${ }^{27}$, et d'autres encore au carrefour de la sociologie, l'histoire et l'anthropologie (Lemieux, 2009); L'interdisciplinarité peut résulter de ce que des linguistes empruntent, par exemple, des concepts à la psychanalyse (Anne-Marie Houdebine, Jacqueline Authier-Revez, Michel Arrivé). C'est d'ailleurs par ce jeu d'emprunts réciproques qu'évoluent les disciplines et que se construisent de nouveaux courants disciplinaires. Autrement dit, ce qui importe pour juger de l'interdisciplinarité est de voir dans quelle mesure un concept né et développé dans une discipline est réutilisé et redéfini ailleurs, dans quelle mesure sont empruntés des outils d'analyse ou dans quelle mesure une discipline a recours à une autre pour interpréter ses résultats, avec effet de retour. Mais ces jeux d'emprunts, d'intégration et d'interdiscursivité doivent être menés en annonçant ce qui est emprunté, intégré ou utilisé, et comment on redéfinit les notions empruntées dans le cadre de sa propre discipline. Rien n'est plus dommageable pour la recherche que le confusionnisme disciplinaire qui se manifeste par 
des emprunts de concepts sans que ceux-ci soient explicités : des emprunts passés sous silence (et donc non repérables), ou des emprunts non redéfinis. Bref, toute utilisation de notions ou hypothèses d'une autre discipline sans interrogations critiques réciproques (Ogien, 2007). Si des sociologues utilisent la notion de grammaire pour, à la suite des propositions de Ludwig Wittgenstein, en faire un concept central dans leur théorisation comme un ensemble de conventions dont les individus auraient une connaissance (implicite ou explicite) qui leur permet d'agir conformément à celles-ci (Boltanski, Thévenot, 1987 ; Lemieux, 2009), il conviendrait que soient rappelées quelles en sont les définitions dans la tradition rhétorique et linguistique, quel aspect peut en être retenu et quelle nouvelle définition on lui attribue. S'agit-il d'un ensemble de règles systémiques comme c'est le cas d'une grammaire de la langue, de normes d'usage plus ou moins ritualisées qui témoignent des manières possibles de dire, ou de conventions réglant les conduites des individus? Si des historiens s'attachent à examiner l'histoire des faits de propagande (Julliard, 2008), il serait utile qu'ils prennent connaissance de ce que peuvent en dire les sociologues qui font des enquêtes de terrain, et des sémiologues et analystes du discours qui mettent en évidence des types d'opinion différents, rarement pris en compte. De même, si des analystes du discours emploient les notions d'idéologie ou de représentation, il est nécessaire qu'ils aillent puiser dans les écrits philosophiques mais aussi dans ceux de la sociologie, de la psychologie sociale et de l'anthropologie, comme je l'ai montré plus haut, afin de se positionner par rapport aux définitions que proposent ces disciplines. À l'inverse, il arrive que les chercheurs d'une discipline retrouvent dans les écrits d'autres disciplines des concepts qui leur sont familiers mais avec d'autres emplois. Par exemple, un analyste du discours retrouvera dans des écrits sociologiques ou de psychologie sociale les concepts de performativité, de pragmatique ou de compétence avec des sens différents de ceux qu'ils ont en sciences du langage; appropriation légitime mais qui nécessiterait une explication quant à la redéfinition qui en est proposée par rapport aux sens originels. De la sorte, on ne déforme pas la notion définie dans l'autre discipline puisque l'on annonce, à la fois, qu'on en comprend bien la définition, qu'on n'en emprunte qu'une partie et qu'on la redéfinit dans sa propre procédure d'analyse. C'est là ce que j'appelle une "interdisciplinarité focalisée ». Car chaque discipline doit rester centrée sur son corps disciplinaire, doit garder son cadre de pertinence qui est ce qui garantit la validité de ses analyses et qui permet que celles-ci puissent être discutées. Toute discipline a besoin d'un lieu géométrique, sans quoi il ne serait pas possible de discuter la pertinence de ses analyses.

\section{Un exemple : l'interdisciplinarité dans l'étude de la communication}

17 Je terminerai en donnant un exemple qui illustre cette interdisciplinarité focalisée à la fois du point de vue des notions, des outils et des résultats. On soutiendra l'hypothèse que tout acte de communication - qu'il s'agisse de communication interpersonnelle privée ou collective publique - se réalise toujours entre un sujet locuteur ${ }^{28}$, et un sujet destinatairerécepteur-interprétant (qui peut devenir à son tour sujet locuteur, de façon symétrique), entre lesquels transite un certain acte de langage porteur de sens ayant une certaine forme ${ }^{29}$. Lorsqu'il s'agit d'une communication collective qui se déploie dans un espace public à l'aide de divers supports de transmission, on dira que la communication se réalise entre une instance de production du langage ${ }^{30}$, et une instance de réception- 
interprétation (qui peut devenir à son tour instance de production mais de façon asymétrique), entre lesquels transite un certain produit porteur de sens ayant diverses formes selon le système de signes (verbal, iconique, gestuel) qui le configure. Ces instances sont constituées par des acteurs qui agissent, qui pensent, qui parlent et qui se trouvent les uns vis-à-vis des autres dans diverses relations selon les statuts et les rôles qu'ils ont à tenir. Certains de ces acteurs tiennent des rôles de producteur de discours (par exemple, pour les médias : direction de l'organe d'information, rédacteurs en chef, journalistes, etc.), d'autres tiennent des rôles de récepteur (par exemple, lecteurs, auditeurs, téléspectateurs), les uns et les autres étant tenus par certaines contraintes tant du côté de la production que du côté de la réception. Du même coup, ces acteurs sont amenés à se représenter la place qu'ils occupent et qu'est censé occuper l'autre, la finalité de l'échange dans laquelle ils sont impliqués et les conditions qui président à cet échange. Une telle hypothèse de fonctionnement de la communication permet de poser un modèle d'analyse distinguant trois lieux de pertinence ${ }^{31}$ : un lieu des conditions de la production, un lieu des conditions de réception et un lieu de réalisation de l'acte de communication comme produit fini. Le premier est celui où sont étudiées les conditions qui surdéterminent ces acteurs : leur identité, leur statut, leurs rôles et les représentations qu'ils se font de l'instance de réception, lesquels témoignent des effets qu'ils cherchent à produire auprès de celle-ci (effets visés). Des travaux de sociologues comme ceux de Cyril Lemieux, Jean-Marie Charon et Rémy Rieffel, pour ne citer que ces chercheurs, se situent dans ce lieu de pertinence. Le deuxième est celui où sont étudiés les processus de compréhension et d'interprétation des acteurs, processus dont on peut penser qu'ils témoignent de la façon dont ces acteurs reçoivent les messages de l'instance de production (effets produits). Les travaux de sociologues (Daniel Dayan, Éric Macé et Éric Maigret, Dominique Mehl, Dominique Pasquier), ou de psychosociologues, bien que de façon fort différente (Odile Camus, Claude Chabrol et Patrice Georget, déjà cités), se situent dans ce lieu de pertinence. Le troisième lieu est celui où sont étudiées les caractéristiques de l'acte de communication dans sa double configuration de forme et de sens, mettant au jour les multiples significations dont il est porteur (effets possibles), car du fait de cette réciprocité non symétrique entre les deux instances de l'échange, le sens de l'acte de communication résulte d'une co-construction opérée par celles-ci ; et donc le sens de tout produit fini est fort des sens issus des effets visés et des effets produits. Les travaux des analystes de discours sur la presse écrite, la radio et la télévision se situent dans ce lieu de pertinence. De la sorte peut s'instaurer une interdisciplinarité aux trois niveaux décrits précédemment. Au niveau des notions, lorsqu'analystes du discours, sociologues et psychosociologues se confrontent à la question des identités sociales et discursives des acteurs de l'échange. Au niveau méthodologique, lorsque le résultat des enquêtes de terrain constituant un nouveau corpus, celui-ci peut être analysé en collaboration entre sociologues et analystes de discours, ou lorsque des psychosociologues utilisent les résultats des analyses discursives (effets possibles) pour en mesurer la compréhension (effets produits) auprès de divers publics par des méthodes expérimentales. Au niveau du recours à d'autres disciplines, lorsque l'on tente d'interpréter ces divers résultats du point de vue d'une perspective historique, ou du point de vue moral en ayant recours à une philosophie de l'éthique. 


\section{Conclusion}

18 Dans les années 70-80, je l'ai déjà dit, Roland Barthes, Pierre Bourdieu, Michel Foucault, Gérard Genette, Edgar Morin, Paul Ricœur et j'en oublie, ont ouvert, en France, la voie à une multiplicité de rapports entre les disciplines des sciences humaines et sociales ${ }^{32}$. Il ne s'agit pas d'un nouveau paradigme, car présupposés épistémologiques et théoriques, méthodes d'analyse et d'interprétations demeurent distincts selon les disciplines et courants disciplinaires. Mais dorénavant, aucune discipline - hors le moment de sa procédure d'analyse - ne peut rester enfermée dans son orthodoxie. Elle doit accepter de s'ouvrir à l'épreuve d'une double interrogation, interne et externe à son champ disciplinaire, et pour ce faire il m'a paru intéressant de proposer cette distinction entre une pluridisciplinarité qui accumule mais n'articule pas, une transdisciplinarité qui traverse les lieux de pertinence mais peut en faire perdre leur horizon, et une interdisciplinarité qui échange, coopère, partage en interrogeant et intégrant de façon critique. Il me semble que c'est dans cette perspective d'interdisciplinarité focalisée que pourront être discutés des concepts et des questions actuellement centrales dans les sciences humaines et sociales: domination, réflexivité, rapport entre démarche empirique et interprétation critique, rapport au savoir, qui débouche sur la question de la posture éthique du chercheur. Mais cela est une autre discussion. Dire que les phénomènes sociaux sont complexes n'est plus une simple formule et dire qu'ils doivent être analysés de façon globale n'est pas seulement un slogan altermondialiste. Mais il ne faudrait pas que cette prise de conscience nous fasse tomber dans trois travers.

19 L'un consisterait penser que seuls de grands systèmes explicatifs permettraient de rendre compte des phénomènes sociaux. Ce serait revenir à la période des grands «-ismes »: les structuralisme, générativisme, interactionnisme ou historicisme d'hier, les sociologisme, économisme et juridisme d'aujourd'hui. Ce serait vouloir essentialiser des modèles d'analyse, au nom de la scientificité, et en les rendant dominants ouvrir la voie à un totalitarisme intellectuel. Un autre serait de penser que le recours simultané - et non critique - à plusieurs disciplines devrait permettre de mieux expliquer les phénomènes. Ce serait pratiquer un amalgame qui pourrait faire illusion mais ne serait que de la poudre aux yeux. Un autre revient à croire que l'on n'arrivera jamais à rendre compte des phénomènes, vu leur complexité, et donc défendre un relativisme neutre accumulant des études empiriques locales sans autre visée que d'apporter un petit caillou à l'édifice d'une cathédrale du savoir dont on ne verrait jamais que quelques pierres. Ce serait-là se retirer de tout désir d'explication. Il est vrai qu'il faut lutter à l'heure actuelle contre une tendance au quantitatisme, quand celui-ci se donne comme seul garant de scientificité, avec ses divers modèles d'analyse statistique, qui voudrait imposer l'idée que c'est dans le calcul mathématique que niche la vérité scientifique, alors que c'est au titre d'outil technologique que celle-ci est utile. Il est également vrai qu'il faut lutter contre le confusionnisme d'une pluridisciplinarité sauvage, ou pour le moins non raisonnée, qui se présenterait comme seul moyen d'appréhender la complexité du monde, et qui occulterait la nécessité de recourir à la rigueur de telle ou telle discipline, seule possibilité de discuter les résultats de façon critique. Il est vrai qu'il faut lutter contre cette autre tendance à se réfugier dans le localisme, au nom d'une certaine authenticité, bien utile lors de la description empirique, mais qui ne permet pas d'avoir une approche critique des phénomènes sociaux. 
20 L'interdisciplinarité focalisée ne doit pas être considérée comme une nouvelle mode pour chercheurs voulant se débarrasser de la rigueur d'une discipline. Elle permet d'échapper à la question si conflictuelle du classement des disciplines en domaines, classements auxquels se sont essayés divers chercheurs parmi lesquels Jean Piaget (1964) et Claude Lévi-Strauss (1964) dans les années 60, Maurice Godelier (1982) et Jean-Claude Passeron (1982) dans les années 90. Exercice fort utile car il oblige à s'interroger sur les fondements des disciplines des sciences humaines et sociales, mais opération qui risque d'occulter le nécessaire travail de confrontation et d'articulation des disciplines entre elles. Au lieu de discuter à l'infini pour savoir quelles sont les sciences humaines et quelles les sciences sociales, au lieu de prendre le risque de démontrer la prédominance de l'une de ces sciences sur les autres, au lieu de tenter un consensus toujours discutable - d'autant que «l'on est loin du consensus minimum ou du compromis provisoire [car] entre les tenants de la science positive, ceux qui défendent des positions herméneutiques et ceux qui refusent l'alternative positivisme/constructivisme, le dialogue est pour le moins difficile et les réponses divergentes " (Boure, 2007 : 317) -, au lieu de cela, une interdisciplinarité focalisée qui n'est pas un modèle mais un état d'esprit, un état d'esprit engendrant une démarche qui cherche à tenir à la fois la multi-appartenance disciplinaire des phénomènes sociaux (interdisciplinarité) et la rigueur d'une discipline (focalisée).

\section{BIBLIOGRAPHIE}

Barthes R., 1984, Essais critiques IV, Paris, Éd. Le Seuil.

Boltanski L., Thévenot L., 1987, De la justification. Les économies de la grandeur, Paris, Presses universitaires de France.

Bonnafous S., Temmar M., éds, 2007, Analyse du discours et sciences humaines et sociales, Paris, éd. Ophrys.

Boure R., 2007, Les sciences humaines et sociales en France, Fernelmont, Éd. EME \& InterCommunications.

Burger M., dir., 2008, L'analyse linguistique du discours des médias : apports, limites et enjeux, Québec, Éd. Nota Bene.

Camus O., 1999, « Les interactions langagières », pp. 259-316, in: Petared P., éd., Psychologie sociale , Paris, Bréal.

Castoriadis C., 1975, L'institution imaginaire de la société, Paris, Éd. Le Seuil.

Chabrol C., 1990, « Réguler la construction de l'identité du sujet du discours », pp. 215-229, in :

Berrendonner A., Parret H., éds, L'interaction communicative, Bern, P. Lang.

Chabrol C., Radu, M., 2009, Psychologie de la communication et persuasion, Bruxelles, De Boeck.

Charaudeau P., dir., 2001, La télévision et la guerre. Déformation ou construction de la réalité ? Le conflit en Bosnie (1990-1994), Bruxelles, Éd. Ina/De Boeck. 
- 2004, «Tiers où es-tu? », pp. 19-41, in : Charaudeau P., dir., La voix cachée du Tiers. Des non-dits du discours, Paris, Éd. L'Harmattan.

- 2007a, «Analyse du discours et communication. L'un dans l'autre ou l'autre dans l'un? ", Semen, 23, pp. 65-77.

- 2007b, «Les stéréotypes, c'est bien, les imaginaires, c'est mieux », pp. 49-63, in : Boyer H., dir., Stéréotypage, stéréotypes : fonctionnement ordinaires et mises en scène, Tome 4, Paris, Éd. L'Harmattan. - dir., 2008a, La médiatisation de la science. Clonage, OGM, Manipulations génétiques, Bruxelles, Éd. Ina/De Boeck.

- 2008b, « La justification d'une approche interdisciplinaire de l'étude des médias », pp. 41-57, in : Burger M., dir., L'analyse linguistique du discours des médias : apports, limites et enjeux, Québec, Éd. Nota Bene.

- dir., 2009, Identités sociales et discursives du sujet parlant, Paris, Éd. L'Harmattan.

Charaudeau P., Maingueneau D., 2005, Dictionnaire d'analyse du discours, Paris, Éd. Le Seuil.

Corcuff Ph., 1995, Les nouvelles sociologies, Paris, Nathan-Université.

Darbellay F., 2005, Interdisciplinarité et transdisciplinarité en analyse des discours. Complexité des textes, intertextualité et transtextualité, Genève, Slatkine.

Debray R., 1994, Manifestes médiologiques, Paris, Gallimard.

Dilthey W., 1883, Einleitung in die Geisteswissenschaften, Stuttgart, Erstausgabe.

Elias N., 1991, La société des individus, trad. de l'allemand par Jeanne Étoré, Paris, Fayard.

Esperet E., 1990, « Apprendre à produire du langage : construction des représentations et processus cognitifs », Le Français dans le monde, nº́cial, pp. 8-15.

Fleury B., Walter J., 2006, « Pour une critique des médias en temps de conflit ? ", Questions de communication, 10, pp. 3-31.

Georget P., 1997, Analyse psycho-socio pragmatique des communications médiatiques à visée persuasive, Thèse de doctorat en psychologie sociale, université Paris 8.

Godelier M., 1982, L'état des sciences de l'homme et de la société en France. Analyse et propositions pour une politique nouvelle, Paris, Éd.La Documentation française.

- 2007, Au fondement des sociétés humaines. Ce que nous apprend l'anthropologie, Paris, A. Michel.

Guilhaumou J., 1993, « à propos de l'analyse du discours : les historiens et le tournant linguistique ", Langage et société, 65, pp. 5-38.

Habermas J., 1981, Théorie de l'agir communicationnel, trad. de l'allemand par J.-M. Ferry, Paris, Fayard.

Houdebine A.-M., dir., 2002, L'imaginaire linguistique, Paris, Éd. L'Harmattan.

Juillard J., 2008, La reine du monde. Essai sur la démocratie d'opinion, Paris, Flammarion.

Jules-Rosette B., 1985, « Entretien avec Harold Garfinkel », Sociétés, 5, sept., pp. 35-39.

Kourilsky F., dir., 1990, Carrefour des sciences, Paris, CNRS Éd.

Lahire B., 2005, L'esprit sociologique, Paris, Éd. La Découverte.

Lefébure P., 2008, Quand les citoyens discutent le lien représentatif, thèse de doctorat en sociologie, Institut d'études politiques, Paris. 
Lemieux C., 2009, Le devoir et la grâce. Pour une analyse grammaticale de l'action, Paris, Éd. Economica.

Lévi-Straus Cl., 1958, Anthropologie structurale, Paris, Plon.

- 1962, La pensée sauvage, Paris, Plon.

- 1964, « Critères scientifiques dans les disciplines sociales et humaines », Revue internationale des sciences sociales, 16, 4, pp. 579-597.

Lochard G., 1999, « Parcours d'un concept dans les études télévisuelles », Hermès, 25, Le dispositif, Paris, éd. cNRS, pp. 143-151.

Miège B., 2000, « Les apports à la recherche des sciences de l'information et de la communication », Réseaux, 100, pp. 547-568.

Ogien A., 2007, Les formes sociales de la pensée. La sociologie après Wittgenstein, Paris, A. Colin.

Passeron J.-C., 1982, « Les sciences sociales : unité et diversité », pp. 10-15, in : Guillaume M., éd., L'état des sciences sociales en France, Paris, éd. La Découverte.

Pêcheux M., 1990, « Les Vérités de la Palice », pp. 175-244, in : Maldidier D., éd., L'inquiétude du discours, Paris, Éd. des Cendres.

Petitclerc A., Schepens Ph., éds, 2009, « Critical Discourse Analysis », Semen, 27, pp. 17-31.

Piaget J., 1964, «Classification des disciplines et connexions interdisciplinaires », Revue internationale des sciences sociales, 16, 4, pp. 598-616.

Plantin C., Doury M., Traverso V., dirs, 2000, Les émotions dans les interactions, Lyon, Presses universitaires de Lyon.

Quéré L., 1999, La sociologie à l'épreuve de l'herméneutique, Paris, Éd. L'Harmattan.

Ricœur P., 1990a, Nouveaux actes sémiotiques, Limoges, Presses de l'université de Limoges.

1990b, Soi-même comme un autre, Paris, Éd. Le Seuil.

Rinn M., 2008, Émotions et discours. L'usage des passions dans la langue, Rennes, Presses universitaires de Rennes.

Robin R., 1973, Histoire et linguistique, Paris, A. Colin.

Rouquette M.-L., Rateau P., 1998, Introduction à l'étude des représentations sociales, Grenoble, Presses universitaires de Grenoble.

Soulages J.-Cl., 2002, « Identités discursives et imaginaires figuratifs », pp. 103-109, in : Houdebine A.-M., dir., L'imaginaire linguistique, Paris, Éd. L'Harmattan.

Touraine A., 2007, « Sociologie sans société », pp. 26-36, in : Wieviorka M., dir., Les sciences sociales en mutation, Auxerre, Éd. Sciences humaines.

Tournier M., 1992, Des mots sur la grève. Propos d'étymologie sociale (1), Paris, Klincksieck.

Trognon A., Ghiglione R., 1993, Où va la pragmatique ?, Grenoble, Presses universitaires de Grenoble.

Wieviorka M., 2007, «Introduction », pp. 9-21, in : Wieviorka M., dir., Les sciences sociales en mutation, Auxerre, Éd. Sciences humaines. 


\section{NOTES}

1. Plus récemment, P. Rosanvallon et J. Rancière.

2. Les sociolinguistes et analystes du discours se souviennent de cette expression qui leur fut attribuée par leurs pairs générativistes.

3. Un certain nombre de programmes de recherche du CNRS associent des chercheurs des sciences biologiques et des sciences de l'information et de la communication autour de certaines controverses scientifiques.

4. Cela se produisit avec certains générativistes qui, dans les années 70 , opposaient à toute critique le : «Mais vous ne pouvez pas comprendre puisque vous n'êtes pas générativiste ».

5. Il ne s'agit que de quelques noms qui représentaient à l'époque des figures de la pensée dans ces différents domaines, mais il va sans dire que c'est à travers les discussions avec tous ceux qui travaillaient autour de ces figures que se construisaient alors réflexions, théories et parfois doctrines. C'est donc à eux que, par la même occasion, je rends ici hommage.

6. Voir sur l'internet le projet du «Campus Condorcet».

7. Appel d'offre du colloque organisé par l'université Charles de Gaulle-Lille 3, en juin 2007.

8. Voir le travail de S. Bonnafous et M. Temmar (2007).

9. Colloque international organisé par l'université Tchavtchadavez (Géorgie) en collaboration avec l'université Paul Valéry-Montpellier 3 (France), en octobre 2007.

10. Colloque international organisé à l'université Nancy 2 les 3 et 4 juin 2010 par le Centre d'études littéraires Jean Mourot et le Centre de recherche sur les médiations.

11. F. Darbellay (2005) met ces deux termes en équivalence. Pour ma part, je préfère les distinguer, appliquant la multidisciplinarité aux cas de rencontres plus globales entre diverses sciences (dures et moins dures) pour s'interroger sur des phénomènes qui peuvent intéresser chacune d'elles.

12. C'est pourquoi ce genre de pensée est difficilement discutable. Il faut la prendre dans sa globalité pour en tirer les meilleurs fruits.

13. Le « sous » n'est pas ici marque d'une infériorité mais, comme on dit en linguistique lexicale, d'une relation co-hyponymique.

14. Le Dictionnaire d'analyse du discours de P. Charaudeau et D. Maingueneau (2005) témoigne de cette diversité.

15. «Constructiviste » n'est pas entendu ici comme s'opposant à ethnométhodologique dans le travail sociologique, mais comme le fait que toute discipline, pour aussi empiriste soit-elle, construit son objet d'analyse.

16. On se rappellera la métaphore de l'« effet Münchhausen » employée par M. Pêcheux (1990) pour signaler l'effet fantasmatique qui fait que le sujet « est interpellé en sujet ».

17. Voir l'historique qu'il en fait dans l'entrée «Analyse du discours» dans le Dictionnaire d'analyse du discours (Charaudeau, Maingueneau, 2002).

18. À part l'acte performatif qui veut que le locuteur ait statut de pouvoir pour que son dire soit un faire. Mais dans la théorie des actes de langage, c'est le seul cas où est prise en compte l'identité sociale du sujet.

19. Cité par B. Jules-Rosette (1985: 35-39).

20. On consultera le Dictionnaire d'analyse du discours (Charaudeau, Maingueneau, 2005) en ses différentes entrées pour observer cette interdisciplinarité de fait.

21. Cette différence est apparue de façon patente, lors d'un atelier de recherche au sein de l'Inathèque, lorsqu'ont été présentées des techniques de recueil de données des écrits circulant sur l'internet par des documentalistes montrant la puissance du modèle à travers des arbres de 
représentation gigantesques, et par des chercheurs de l'EHESS ayant soumis leur instrumentation à des hypothèses de recherche.

22. Je fais ici allusion à un travail qui fut mené au sein du CAD sur le discours publicitaire : une expérimentation fut montée à partir de catégories énonciatives issues d'une analyse d'un corpus publicitaire, pour vérifier les perceptions et reconnaissances de ces catégories et les attitudes appréciatives, recherche menée conjointement par J.-Cl. Soulages et $\mathrm{Cl}$. Chabrol. Voir également le travail sur les « focus group » de P. Lefébure (2008) ainsi que les travaux d'O. Camus (1999) et P. Georget (1997).

23. Voir particulièrement, du point de vue de la sociologie, L. Quéré (1999) et la discussion plus générale qu'en propose A. Ogien (2007).

24. Voir à ce propos la revue Critique, 579/580, août-sept. 1995, dans laquelle des philosophes parlent de P. Bourdieu et de la philosophie.

25. Charte du Campus Condorcet consultable sur l'internet.

26. C'est le cas de certaines contributions de l'ouvrage de S. Bonnafous et M. Temmar (2007).

27. Voir le Programme pRotéE (Programme de recherche et d'opérationnalisation sur les topiques de l'éthique et de l'équivalence) animé par L. Boltanski et L. Thévenot depuis les années 80 .

28. «Locuteur » est entendu ici au sens générique de tout sujet produisant un acte de langage oral ou écrit.

29. En réalité, le schéma est plus complexe du fait d'un dédoublement des sujets du langage en identité sociale et discursive. Voir l'entrée «Sujet parlant » du Dictionnaire d'analyse du discours (Charaudeau, Maingueneau, 2005).

30. «Langage » est ici entendu dans un sens large comme tout système de signes permettant de communiquer.

31. Voir l'article de P. Charaudeau (2008b).

32. Ce pourquoi il est nécessaire de maintenir conjointement « humaines » et « sociales».

\section{RÉSUMÉS}

À une époque où il est beaucoup question de pluridisciplinarité ou d'interdisciplinarité alors que, parallèlement, le système institutionnel et une certaine corporation de chercheurs ne prennent en considération que des études monodisciplinaires technicistes au nom de la scientificité, je propose d'interroger la notion même d'interdisciplinarité à la lumière de certains travaux qui s'en réclament. La question est abordée par le biais de quelques problématisations qui sont susceptibles de rendre compte de la façon dont peuvent être décrits les phénomènes sociaux à travers certaines pratiques analysantes. M'interrogeant d'abord sur ce qui constitue une discipline, je voudrais tenter de pointer, en tant que sémiologue et analyste du discours, ce que devraient être les conditions d'une interdisciplinarité, et montrer comment celle-ci peut être mise en œuvre dans le champ de l'analyse des phénomènes de communication.

INDEX

Mots-clés : analyse du discours, communication, identité, interdisciplinarité, pluridisciplinarité, sciences humaines et sociales, sujet, représentations 


\section{AUTEUR}

\section{PATRICK CHARAUDEAU}

Laboratoire Communication et politique, CNRS

Université Paris 13

patrick.charaudeau@free.fr 